\title{
Comparison of prompt-fission neutron multiplicities and energy spectra for intermediate energy proton-and neutron-induced fission
}

\author{
Oleg Batenkov¹,a, Georgy Boikov${ }^{1}$, Vilen Eismont ${ }^{1}$, Mikhail Majorov${ }^{1}$, Sergey Soloviev¹, Jan Blomgren ${ }^{2}$, \\ and Walter Loveland ${ }^{3}$ \\ 1 V.G. Khlopin Radium Institute, 2oi Murinskiy Prospect 28, Saint-Petersburg 194021, Russia \\ 2 Department of Neutron Research, Uppsala University, Box 525, 75120 Uppsala, Sweden \\ 3 Oregon State University, USA
}

\begin{abstract}
The number and spectra of neutrons, in particular the number and spectra of prompt neutrons from the fission of actinides, at the intermediate energies represent important physical quantities, which determine the possibility of applying of one or another design of ADS. The multiplicities and energy distributions of prompt neutrons in interactions of ${ }^{232} \mathrm{Th},{ }^{235,238} \mathrm{U}$ and ${ }^{237} \mathrm{~Np}$ with 50 and $96 \mathrm{MeV}$ protons were measured by our group. The data are compared with results of recent measurements of the average multiplicities and spectra of prompt neutrons at the fission of ${ }^{235,238} \mathrm{U}$ induced by neutrons of the same energies. The correlation of the measured characteristics with neutron-proton compositions of composite nuclei is discussed. The similarity of characteristics for fission induced by neutrons and protons is noted.
\end{abstract}

\section{Introduction}

The number and spectra of prompt neutrons from the fission of actinides, at intermediate energies, determines the applicability of different variants of ADS designs. At the same time a knowledge of these characteristics is of great importance for theory because it leads to a better understanding of the mechanism of the interaction of fast neutrons with nuclei and properties of the fission of heated nuclei-the dynamics of the sharing of energy brought in into a nucleus by an incident nucleon between the collective and single-particle degrees of freedom, features of the de-excitation of high excited states of fission fragments. But numerous measurements, which have been made, were carried out for a restricted energy range of incident neutrons- i.e., nuclear reactor energies $(\mathrm{E}<20 \mathrm{MeV})$. Only recently, have results of measurements of the average multiplicity of prompt neutrons of the fission of ${ }^{238} \mathrm{U}$ and ${ }^{235} U$ [1] and spectra of such neutrons for ${ }^{238} U$ [2] carried out at incident nucleon energies from 0.7 to $200 \mathrm{MeV}$ been published. It is supposed that significant, additional, information on the energy dependence of characteristics of fission may be obtained in more easily performed measurements using proton beams (the intensity of proton beams is higher than the intensity of neutron beams by some orders of magnitude). For intermediate energy protons only data for $\mathrm{E}<60 \mathrm{MeV}$ for ${ }^{238} \mathrm{U}$ [3] and $\mathrm{E}=155 \mathrm{MeV}$ for ${ }^{238} \mathrm{U}$ [4] exist. Moreover, in the latter work published in 1970 only average multiplicities were measured. The present work is intended to fill the gap using modern experimental techniques, in the range of investigated nuclei and to compare results of measurements with protons and neutrons.

\section{Experimental apparatus and techniques}

The experimental apparatus and techniques used to make this measurement are very similar to that described previously [5].

\footnotetext{
a Presenting author, e-mail: obatenkov@atom.nw.ru
}

In this study, beams of 50 and $96 \mathrm{MeV}$ protons from the Gustav Werner synchrocyclotron at the T. Svedberg Laboratory, Uppsala University, Uppsala, Sweden were used to irradiate targets of ${ }^{232} \mathrm{Th},{ }^{235} \mathrm{U},{ }^{238} \mathrm{U}$ and ${ }^{237} \mathrm{~Np}$. The actinide targets were mounted in the center of a thin-walled $(0.8 \mathrm{~mm})$ stainless steel chamber. (The time structure of the proton beam involves a $3 \mathrm{~ns}$ wide bunch with $70 \mathrm{~ns}$ between bunches.) The targets were $100-320 \mu \mathrm{g} / \mathrm{cm}^{2}$ of $\mathrm{ThF}_{4}, \mathrm{UF}_{4}$ and $\mathrm{NpO}_{2}$ evaporated onto a $60 \mu \mathrm{g} / \mathrm{cm}^{2} \mathrm{Al}_{2} \mathrm{O}_{3}$ backing and covered by $20 \mu \mathrm{g} / \mathrm{cm}^{2} \mathrm{Au}$. After passing through the scattering chamber, the beam was stopped in a well-shielded Faraday cup $\sim 25 \mathrm{~m}$ downstream of the target.
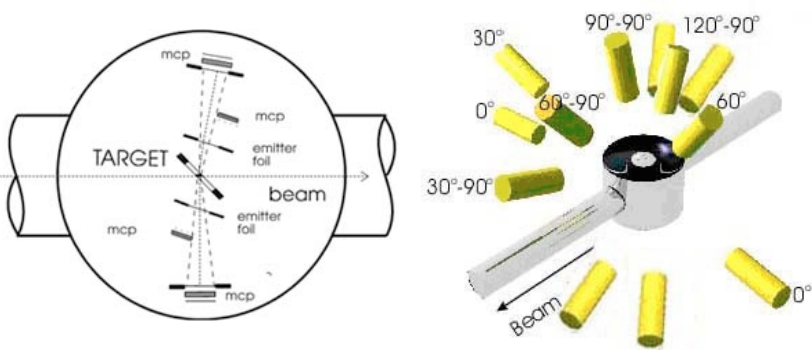

Fig. 1. Experimental arrangement. The location of the neutron detectors are shown in the right panel, and a schematic drawing of the scattering chamber is shown in the left panel.

In figure 1, we show a schematic diagram of the arrangement of the fission detectors inside the chamber and the placement of the twelve stilbene neutron detectors used in this study. The fission detectors consisted of two time-offlight telescopes mounted at $+80^{\circ}$ and $-90^{\circ}$ with respect to the incident proton beam. Start and stop signals for the time-offlight detectors were obtained from microchannel plate (MCP) detectors. The fission fragments passed through $60 \mu \mathrm{g} / \mathrm{cm}^{2}$ $\mathrm{Al}_{2} \mathrm{O}_{3}$ emitter foils, knocking out a shower of electrons that 
are detected in the MCPs. The time resolution of each telescope was $\sim 80 \mathrm{ps}$ with a fragment registration efficiency of $98 \%$. For each fission event, the velocities of both fragments were measured and assuming, the mass number of the fissioning system was $\left(\mathrm{A}+1-v_{\text {prefission }}\right)$ where the mass number of the target nucleus is $\mathrm{A}$ and $v_{\text {prefission }}$ is the average number of pre-fission neutrons, the fragment masses were calculated on an event-by-event basis.

The energy of any neutrons associated with each fission event was measured using time-of-flight methods. The stop signal for the neutron time-of-flight was taken to be the fissionfission coincidence with the start signal being from the stilbene neutron detectors. Typical time resolutions of $\sim 1 \mathrm{~ns}$ were obtained with neutron flight paths of $46-50 \mathrm{~cm}$. This corresponds to an energy resolution for $2 \mathrm{MeV}$ neutrons of $\sim 9 \%$. Neutrons were separated from $\gamma$-rays using pulse shape discrimination. The $\mathrm{n} / \gamma$ separation was $>10^{4}$ for $\mathrm{E}_{n}>0.5 \mathrm{MeV}$.

The efficiency of the neutron detectors for $\mathrm{E}_{n}<9 \mathrm{MeV}$ was defined as the ratio of the measured neutron spectrum from ${ }^{252} \mathrm{Cf}$ spontaneous fission to the known distribution. Calibration spectra using ${ }^{252} \mathrm{Cf}$ were measured before and after each experiment by placing a small ionization chamber containing ${ }^{252} \mathrm{Cf}$ in the target position. For $\mathrm{E}_{n}>9 \mathrm{MeV}$, the detector efficiencies were calculated.

Neutron detectors were at angles of $0,30,54,60$, and $90^{\circ}$ with respect to the direction of motion of the fission fragments. Detectors were also at angles of 30, 36, 50, 60, 90, 120 , and $144^{\circ}$ with respect to the proton beams. As described earlier, the neutron flight paths were $46-50 \mathrm{~cm}$ with detector diameters of $5-7 \mathrm{~cm}$ and with detector thicknesses of $2-3 \mathrm{~cm}$. The time-of-flight distribution of background events was obtained for each detector from gating on a subsequent beam burst. The number of fission-neutron events collected for each energy for each target was $\sim 10^{6}$, with typical proton beam currents of $15 \mathrm{nA}$.

\section{Data analysis and results}

For each beam-target combination, the experimental results of these measurements are a series of neutron time-of-flight spectra, measured at twelve different angles and their associated fission fragment distributions. (The data are collected on an event-by-event basis with each event including time-of-flights of the emitted neutron and the two fission fragments.)

In figure 2, we show some representative neutron timeof-flight spectra for the interaction of $96 \mathrm{MeV}$ protons with ${ }^{235} \mathrm{U}$ along with background spectra and calibration spectra taken with ${ }^{252} \mathrm{Cf}$. These time-of-flight spectra were converted into neutron energy spectra. In figure 3, we show some representative fission-associated neutron spectra for the reaction of $96 \mathrm{MeV}$ protons with ${ }^{235} \mathrm{U}$ at fission-neutron angles of 0 and $87^{\circ}$ (beam-neutron angle of $90^{\circ}$ ), while in figure 3 we show typical fission-associated neutron spectra at beamneutron angles of 30, 50, 90, and $120^{\circ}$ (at fission-neutron angles $\left.\sim 90^{\circ}\right)$. As expected, the high energy neutrons $\left(\mathrm{E}_{n}>\right.$ $15 \mathrm{MeV}$ ) from direct processes are strongly forwardly peaked with respect to the beam axis. Also the angular distributions of the neutrons with respect to the fragment direction of motion

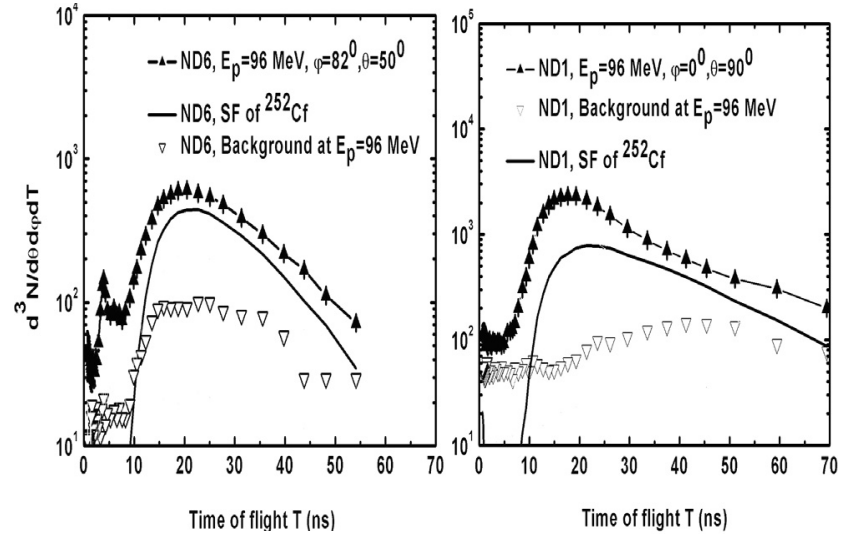

Fig. 2. Representative time-of-flight spectra for the interaction of $96 \mathrm{MeV}$ protons with ${ }^{235} \mathrm{U}$.

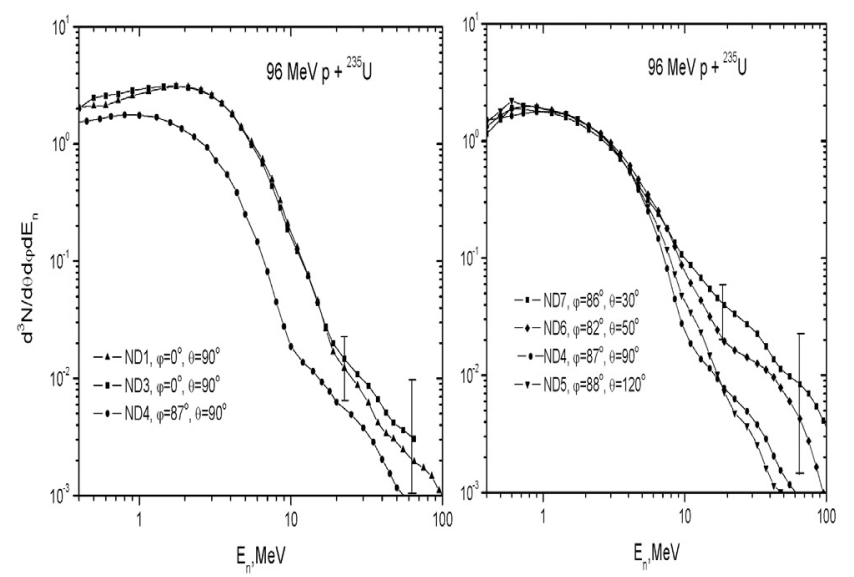

Fig. 3. Representative neutron energy spectra for the interaction of $96 \mathrm{MeV}$ protons with ${ }^{235} \mathrm{U}$.

are forward-peaked with the higher energy neutrons being more isotropic.

The number of neutrons emitted per fission, $v$, for the $50 \mathrm{MeV}$ proton induced fission of ${ }^{232} \mathrm{Th},{ }^{235} \mathrm{U},{ }^{238} \mathrm{U}$ and ${ }^{237} \mathrm{~Np}$ is $7.35 \pm 0.07,6.86 \pm 0.06,7.44 \pm 0.07$, and $7.24 \pm 0.06$ while the same quantities for the $96 \mathrm{MeV}$ proton induced fission are $9.17 \pm 0.10,8.57 \pm 0.08,9.27 \pm 0.08$, and $8.58 \pm$ 0.1 . The average neutron energy in the laboratory system for the $50 \mathrm{MeV}$ proton induced fission of ${ }^{232} \mathrm{Th},{ }^{235} \mathrm{U},{ }^{238} \mathrm{U}$ and ${ }^{237} \mathrm{~Np}$ is $2.63 \pm 0.02,2.77 \pm 0.02,2.72 \pm 0.02,2.83 \pm$ $0.02 \mathrm{MeV}$ while the same quantities for the $96 \mathrm{MeV}$ proton induced reaction are $3.09 \pm 0.04,3.09 \pm 0.03,3.12 \pm 0.03$, and $3.12 \pm 0.04 \mathrm{MeV}$, respectively. The average neutron energies in the laboratory system are largely independent of the target nucleus and increase with the beam energy. (Typical average laboratory neutron energies in the low energy neutron induced fission of these nuclei are about $2.0 \mathrm{MeV}$.)

In figure 4, we show, for typical projectile energy-target system, the decomposition of the neutron energy spectra into three components: (a) pre-equilibrium neutrons emitted in the initial nucleon-nucleus interaction, consisting of two stages, an intranuclear cascade in which the primary particles are re-scattered several times before absorption or leaving the nucleus and a second "exciton" stage where conventional 

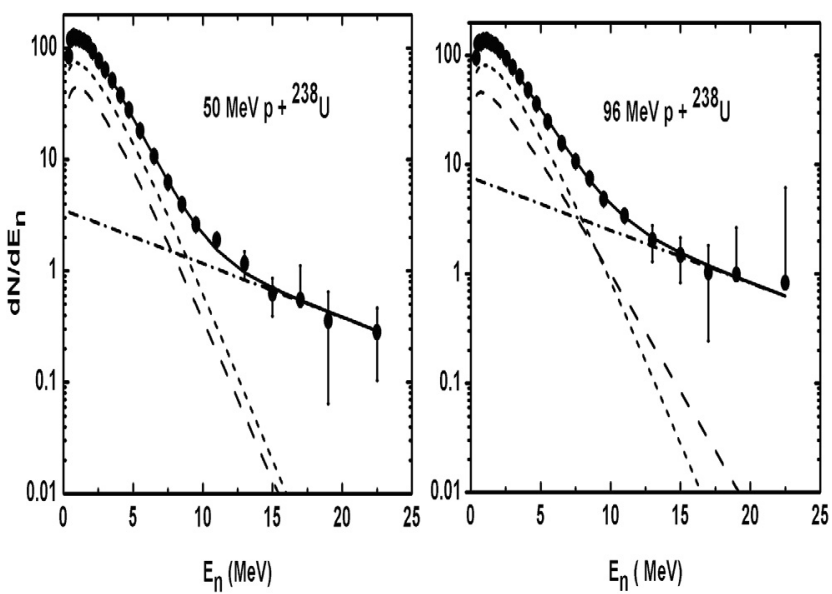

Fig. 4. Decomposition of the neutron energy spectra into their components. Solid lines and symbols indicate the overall spectra, the dot-dashed line the pre-equilbrium component, the short dashed line the post fission component and the long dashed line the equilibrium component.

pre-equilibrium emission takes place (dot-dashed line), (b) equilibrium neutrons emitted by the equilibrated nucleus prior to fission (long dashed line) and (c) post fission neutrons emitted by the fully accelerated fission fragments (short dashed line). As remarked earlier, the so-called scission neutrons and neutron emitted by the accelerating fragments are grouped into the "equilibrium component".

To do this decomposition, one begins by removing the preequilibrium component from each angle resolved spectrum. This is done by assuming that all neutrons above $15 \mathrm{MeV}$ are due to pre-equilibrium processes and fitting the energy spectra with an equation of the form

$$
\mathrm{dN} / \mathrm{dE}_{\mathrm{n}}=v_{\text {pre }-1} \operatorname{Aexp}\left[-\left(\mathrm{E}_{\mathrm{n}}-\mathrm{C}\right) / \mathrm{T}\right)
$$

where $\mathrm{A}$ and $\mathrm{C}$ are normalizing constants and $\mathrm{T}$ is a slope parameter giving the slope of the pre-equilibrium energy distribution. The slope parameter $\mathrm{T}$ was found to be $9 \mathrm{MeV}^{-1}$ for all systems. The values of $v_{\text {pre-1 }}$ are tabulated in table 1 along with the average energy (in the rest frame of the emitting system) of each pre-equilibrium neutron. This component does not depend on the nature of the target nucleus and only weakly depends on the beam energy. (The additional energy carried away by these pre-equilibrium neutrons only increases only about $4.5 \mathrm{MeV}$ as the beam energy increases $46 \mathrm{MeV}$.) While the pre-equilibrium neutrons include the most energetic neutrons, these high energy neutrons are very few in number making their contributions to the energetics relatively small.

Once the pre-equilbrium neutrons have been removed from the spectra, the components due to the equilibrium and post-fission neutrons are resolved. In a first approximation, all neutrons emitted at $0^{\circ}$ with respect to the fragment direction of motion are assumed to be due to post fission neutrons while all neutrons emitted at $90^{\circ}$ are assumed to be equilibrium neutrons. The energy spectra in the rest frame of the emitting nucleus of these two components are fitted using the equations

$$
\mathrm{dN} / \mathrm{dE}_{\mathrm{n}}=v_{\text {post }} \mathrm{B} \mathrm{E}_{\mathrm{n}}{ }^{\mathrm{b}} \exp \left[-\mathrm{E}_{\mathrm{n}} / \mathrm{T}_{\text {post }}\right]
$$

Table 1. The average energy (in the rest frame of the emitting system) and number of neutrons emitted in pre-equilibrium processes, by the

\begin{tabular}{|c|c|c|c|c|}
\hline Target & \multicolumn{2}{|c|}{$\mathrm{E}_{\mathrm{p}}=50 \mathrm{MeV}$} & \multicolumn{2}{|c|}{$\mathrm{E}_{\mathrm{p}}=96 \mathrm{MeV}$} \\
\hline & $v_{\text {pre-1 }}$ & $\left\langle\mathrm{E}_{\mathrm{n}}\right\rangle(\mathrm{MeV})$ & $v_{\text {pre-1 }}$ & $\left\langle\mathrm{E}_{\mathrm{n}}\right\rangle(\mathrm{MeV})$ \\
\hline${ }^{232} \mathrm{Th}$ & $0.5 \pm 0.3$ & $8.8 \pm 1.0$ & $1.1 \pm 0.3$ & $9.1 \pm 1.0$ \\
\hline${ }^{235} \mathrm{U}$ & $0.5 \pm 0.3$ & $8.8 \pm 1.0$ & $1.1 \pm 0.3$ & $9.1 \pm 1.0$ \\
\hline${ }^{238} \mathrm{U}$ & $0.5 \pm 0.3$ & $8.8 \pm 1.0$ & $1.1 \pm 0.3$ & $9.1 \pm 1.0$ \\
\hline${ }^{237} \mathrm{~Np}$ & $0.5 \pm 0.3$ & $8.8 \pm 1.0$ & $1.0 \pm 0.3$ & $9.1 \pm 1.0$ \\
\hline Target & \multicolumn{2}{|c|}{$E_{p}=50 \mathrm{MeV}$} & \multicolumn{2}{|c|}{$\mathrm{E}_{\mathrm{p}}=96 \mathrm{MeV}$} \\
\hline${ }^{232} \mathrm{Th}$ & $4.4 \pm 0.3$ & $1.65 \pm 0.03$ & $4.9 \pm 0.3$ & $1.76 \pm 1.0$ \\
\hline${ }^{235} \mathrm{U}$ & $4.9 \pm 0.3$ & $1.74 \pm 0.03$ & $5.0 \pm 0.3$ & $1.76 \pm 1.0$ \\
\hline${ }^{238} \mathrm{U}$ & $4.4 \pm 0.3$ & $1.65 \pm 0.03$ & $5.1 \pm 0.3$ & $1.76 \pm 1.0$ \\
\hline${ }^{237} \mathrm{~Np}$ & $4.8 \pm 0.3$ & $1.76 \pm 0.03$ & $4.9 \pm 0.3$ & $1.76 \pm 1.0$ \\
\hline Target & \multicolumn{2}{|c|}{$\mathrm{E}_{\mathrm{p}}=50 \mathrm{MeV}$} & \multicolumn{2}{|c|}{$\mathrm{E}_{\mathrm{p}}=96 \mathrm{MeV}$} \\
\hline & $v_{\mathrm{eq}}$ & $\left\langle\mathrm{E}_{\mathrm{n}}\right\rangle(\mathrm{MeV})$ & $v_{\mathrm{eq}}$ & $\left\langle\mathrm{E}_{\mathrm{n}}\right\rangle(\mathrm{MeV})$ \\
\hline${ }^{232} \mathrm{Th}$ & $2.4 \pm 0.3$ & $2.30 \pm 0.03$ & $3.4 \pm 0.3$ & $2.60 \pm 1.0$ \\
\hline${ }^{235} \mathrm{U}$ & $1.5 \pm 0.3$ & $2.50 \pm 0.03$ & $2.7 \pm 0.3$ & $2.70 \pm 1.0$ \\
\hline${ }^{238} \mathrm{U}$ & $2.6 \pm 0.3$ & $2.40 \pm 0.03$ & $2.9 \pm 0.3$ & $2.70 \pm 1.0$ \\
\hline${ }^{237} \mathrm{~Np}$ & $1.9 \pm 0.3$ & $2.40 \pm 0.03$ & $3.0 \pm 0.3$ & $2.90 \pm 1.0$ \\
\hline
\end{tabular}
fully accelerated fission fragments and prior to fission.

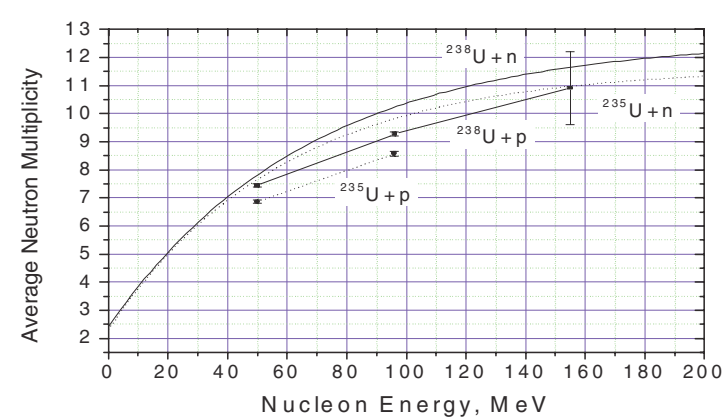

Fig. 5. Prompt fission-neutron average multiplicity for ${ }^{235} \mathrm{U}$ and ${ }^{238} \mathrm{U}$ as a function of incident nucleon energy, the approximating functions - for neutrons (solid line - ${ }^{238} \mathrm{U}$, dashed line - ${ }^{235} \mathrm{U}$ ) [1], points - for protons (solid square connected by a solid line - for ${ }^{238} \mathrm{U}$ including data for $\mathrm{E}=155 \mathrm{MeV}$ from [4], solid circles connected by a dashed line - for $\left.{ }^{235} \mathrm{U}\right)$.

$$
\mathrm{dN} / \mathrm{dE}_{\mathrm{n}}=v_{\mathrm{eq}} \mathrm{DE} \mathrm{E}_{\mathrm{n}}{ }^{\mathrm{d}} \exp \left[-\mathrm{E}_{\mathrm{n}} / \mathrm{T}_{\mathrm{cn}}\right]
$$

where the constants $\mathrm{B}, \mathrm{D}, \mathrm{b}, \mathrm{d}, \mathrm{T}_{\text {post }}$ and $\mathrm{T}_{c n}$ are determined in the fitting process. Following the determination of these constants, they are used with the above equations to fit, in an iterative manner, the angle-resolved energy spectra to give the values of $v_{\text {post }}$, the post scission neutron multiplicity per fission event and $v_{\text {eq }}$, the multiplicity of equilibrium neutrons emitted per fission event. The values of these quantities are tabulated in table 1.

\section{Discussion}

In figure 5 our data on the average multiplicity of neutrons, $\left\langle v_{\mathrm{p}}\right\rangle$ produced in the fission of ${ }^{235} \mathrm{U}$ and ${ }^{238} \mathrm{U}$ are compared with fitting functions for these values obtained in [1] (for the same energy region of fission-neutrons).

It is seen that the energy dependence of the average multiplicity for the $(p, f)$ and $(n, f)$ reactions looks similar. 


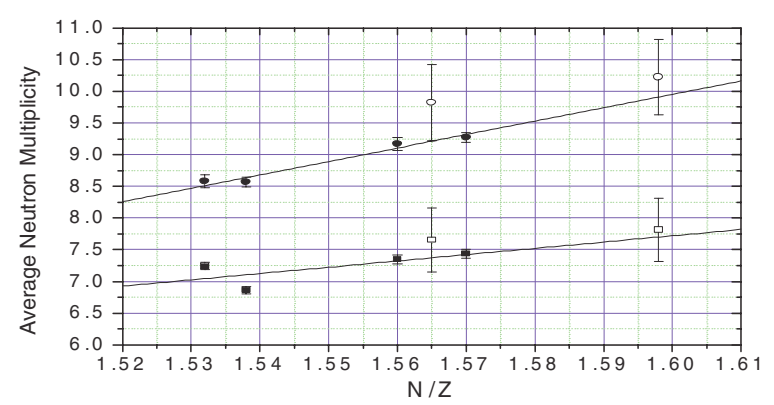

Fig. 6. Prompt fission-neutron average multiplicity for systems ${ }^{237} \mathrm{~Np}+\mathrm{p},{ }^{235} \mathrm{U}+\mathrm{p},{ }^{232} \mathrm{Th}+\mathrm{p},{ }^{235} \mathrm{U}+\mathrm{n},{ }^{238} \mathrm{U}+\mathrm{p}$ and ${ }^{238} \mathrm{U}+\mathrm{n}$ as a function of the ratio of the number of protons to the number neutrons for composite nuclei (systems are listed by way of N/Z). Open symbols are for neutron-induced reactions.

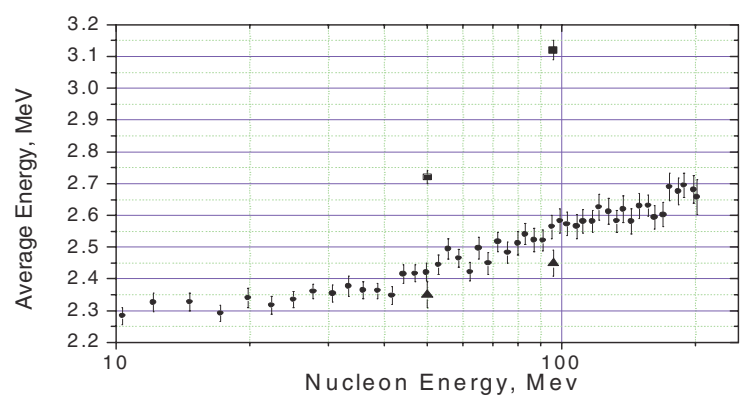

Fig. 7. Prompt fission-neutron average energy for ${ }^{238} \mathrm{U}$ as a function of incident nucleon energy: circles - neutrons [2], squares - for protons for the range of fission neutrons 0.4 to $22.5 \mathrm{MeV}$, triangles - for protons for the range of fission neutrons $0.65-7.5 \mathrm{MeV}$.

All points for ${ }^{238} \mathrm{U}(\mathrm{p}, \mathrm{f})$ including the point at $155 \mathrm{MeV}$ [4] and for ${ }^{235} \mathrm{U}(\mathrm{p}, \mathrm{f})$ lie lower than the corresponding $(\mathrm{n}, \mathrm{f})$ reaction points. As in the case of reactions with neutrons [1] $\left.\left\langle v_{\mathrm{p}}\right\rangle\left({ }^{238} U\right)\right\rangle\left\langle v_{\mathrm{p}}\right\rangle\left({ }^{235} \mathrm{U}\right)$. The comparison of data on ${ }^{235} \mathrm{U}$ and ${ }^{238} \mathrm{U}$ with data on other nuclides demonstrates an increase of $\left\langle v_{\mathrm{p}}\right\rangle$ with the increase of $\mathrm{N} / \mathrm{Z}$ (where $\mathrm{N}$ and $\mathrm{Z}$ numbers of neutrons and protons in composite nuclei). The slope of $\left\langle v_{\mathrm{p}}\right\rangle$ increases with incident nucleon energy.

This is illustrated in figure 6 , which includes both data on protons and on neutrons.

It may be noted that the similarity of energy dependence and the absolute values of $\left\langle v_{\mathrm{p}}\right\rangle$ also occurs in the comparison of the average energies of prompt neutrons at the neutron and proton induced fission of ${ }^{238} \mathrm{U}$. This may be seen in figure 7 , where together with data from [2], our results on the average energies of spectra of all detected neutrons including cascade neutrons with energies up to $22.5 \mathrm{MeV}$ and spectra "deduced" from the latter, are presented.

The authors of [2] found that at energies higher than $50 \mathrm{MeV}$ the average energy of fission-neutrons in the laboratory frame slightly increases with the energy of incident neutrons due to both the rise of energy in a center-of mass frame (CMF) and the rise of the kinetic energy of fission fragments. We suppose that this may be a result of the rise of the excitation energy of fission fragments that in turn leads to the rise of the temperature of neutron spectra in CMF and to a more early emission of neutrons from fragments - up to the moment of the beginning of acceleration that increases the detected kinetic energy of fission fragments. The effect of emitting neutrons from fission fragments before the stage of being fully accelerated was treated in [7].

\section{Conclusion}

The similarity of fission properties in $(p, f)$ and $(n, f)$ reactions is considered to be the result of the effect of the "compound nucleus". As calculations made with contemporary codes TALYS [9,10] and CEM03 [11,12] show, this effect is a consequence of the similar characteristics of the intermediate compound nuclei, formed after a intra-nuclear cascade and pre-equilibrium emission. Due to the fact that intensities of protons beams are some orders of magnitude higher than intensities of neutron beams, the possibility of investigating, in detail, the dependence of the characteristics of fissionneutrons on the excitation energy of a nucleus, including determining various differential characteristics, correlations with fission fragments masses and kinetic energies, for a wide circle of actinides becomes more realistic. The investigation of such correlations is necessary for the development of our understanding of such a multi-parameter process as fission to apply the process in nuclear plants using intermediate energy neutrons.

\section{References}

1. Th. Ethvignot et al., in Proc. Int. Conf. on Nuclear Data for Science and Technology, Santa Fe, NM, Sept. 26-Oct. 1, 2004, AIP Conf. Proc. 769 (2005), p. 656.

2. Th. Ethvignot et. al., Phys. Lett. B 575, 221 (2003).

3. V.A. Rubchenya et al., Nucl. Instrum. Meth. Phys. Res. A 463, 653 (2001).

4. E. Cheifetz et al., Phys. Rev. C 2, 256 (1970).

5. O. Batenkov et al., Nucl. Instrum. Meth. Phys. Res. A 394, 235 (1997).

6. W. Mannhart, IAEA Tecdoc-410, 158 (1986).

7. V.P. Eismont, At. Energy 19, 133 (1965).

8. A.N. Smirnov et al. (these proceedings).

9. A.J. Koning et al., in Proc. Int. Conf. on Nuclear Data for Science and Technology, Santa Fe, NM, Sept. 26-Oct. 1, 2004, AIP Conf. Proc. 769 (2005), p. 1154.

10. A.N. Smirnov et al., ibid., p. 637.

11. S.G. Mashnik et al., LANL Report LA-UR-05-2686, Los Alamos, 2005; E-prints: nucl-th/0502019; nucl-th/0503061.

12. K.K. Gudima (private communication). 